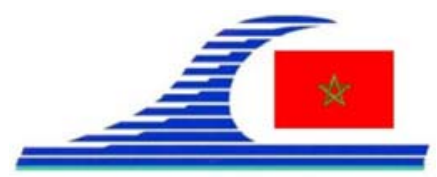

Conférence Méditerranéenne Côtière et Maritime

EDITION 2, TANGER, MAROC (2011)

Coastal and Maritime Mediterranean Conference

Disponible en ligne - http://www.paralia.fr - Available online

\title{
Evolution du trait de côte de Gabès-oued Ferd (SE de la Tunisie)
}

\author{
Ikram BARDI ${ }^{1}$, Saadi ABDELJAOUAD ${ }^{1}$, Mongi SOUAYED ${ }^{1}$
}

1. Département de Géologie, Fac. des Sc. de Tunis, Univ. de Tunis-El-Manar, Tunisie.

Laboratoire des Ressources Minérales et Environnement, F.S.Tunis, Tunisie.

ikram.bardi@yahoo.fr

\section{Résumé :}

La partie sud du golfe de Gabès, au SE de la Tunisie, est une côte sableuse de type microtidale, soumise à un climat semi-aride. Il s'y développe actuellement une succession de flèches sableuses abritant des lagunes. Cette frange littorale est alimentée en sable fin d'origine fluviale par les oueds Gabès, Sourrag et Ferd, en période de fortes crues alimentées par des pluies torrentielles. On cherchera donc à déterminer l'évolution du trait de côte au cours des dernières décennies et à établir les conditions hydrodynamiques et anthropiques ayant favorisé cette évolution. Pour ce faire, on s'est appuyé sur l'étude diachronique de la ligne de rivage à partir des photographies aériennes datant de 1963, 1985 et 1997.

Ainsi, l'évolution de cette côte est assujettie à des variations continues qui témoignent une dynamique sédimentaire active.

\section{Mots clés :}

Côte sableuse - Evolution du trait de côte - Dynamique sédimentaire

\section{Introduction}

Le long de la côte sud du golfe de Gabès (SE de la Tunisie), qui s'étire sur environ 20 km entre le port de Gabès et l'Oued Ferd, se développe actuellement une succession de flèches sableuses de quelques kilomètres de longueur, abritant des lagunes (Fig. 1). Il s'agit d'un milieu microtidal où le marnage atteint exceptionnellement 2,3 m pendant les périodes de vives eaux. Les sédiments le long du littoral montrent une granulométrie moyenne à fine variant entre $350 \mu \mathrm{m}$ à $100 \mu \mathrm{m}$. cette côte est soumise à des houles des secteurs variés, celles du N à NNE génèrent des courants de dérive littorale de direction NW-SE; alors que les houles de directions $\mathrm{E}$ à SE, produisent une dérive littorale de direction SE-NW (Fig. 1). Ces courants sont mis en évidence par le système de modélisation côtière (BARDI, 2010). D'autre part, la partie nord de ce littoral est marquée par l'aménagement d'un port dans les années soixante dix qui a modifié l'allure du trait de côte en face de la ville de Gabès (Fig. 2). Dans ce travail nous analysons l'évolution du trait de côte de Gabès-oued Ferd, au moyen d'une analyse diachronique entre 1963 et 1997 et nous précisons les causes de cette évolution. 
La connaissance de la Mer :

un vecteur du développement durable en Méditerranée

\section{Matériels et méthodes d'analyses}

Le travail d'analyse est basé sur l'évolution spatio-temporelle du trait de côte, à partir de photographies aériennes datant de 1963, 1985, et 1997, couvrant la zone d'étude, sous projection Lambert Sud Tunisien. La cartographie du trait de côte a été réalisée au sein de l'Office Tunisien de la Topographie et Cartographie (O.T.C.) par le traitement photogrammétrique. Sur les littoraux microtidaux le trait de côte est facilement identifiable sur les photographies aériennes, par la différence radiométrique entre les parties sèches et humides de l'estran (DOLAN et al., 1978). Dans le golfe de Gabès le marnage est relativement important en période de vives eaux, dans ce cas la ligne de rivage n'est pas un indicateur stable puisqu'elle se déplace sur l'estran avec la marée. De plus, pour les 3 missions de photographies aériennes nous n'avons pas bénéficié des données concernant le moment des prises de vue (marée de vives eaux ou de mortes eaux). Pour ces faits, le trait de côte s’est porté sur la limite de végétation, indicateur le plus approprié pour observer et quantifier la mobilité du rivage (THIELER \& DANFORTH, 1994). Ainsi, nous superposons par la suite les 3 lignes de côte géoreférencées, et nous pouvons déterminer de ce fait la variation du rivage.

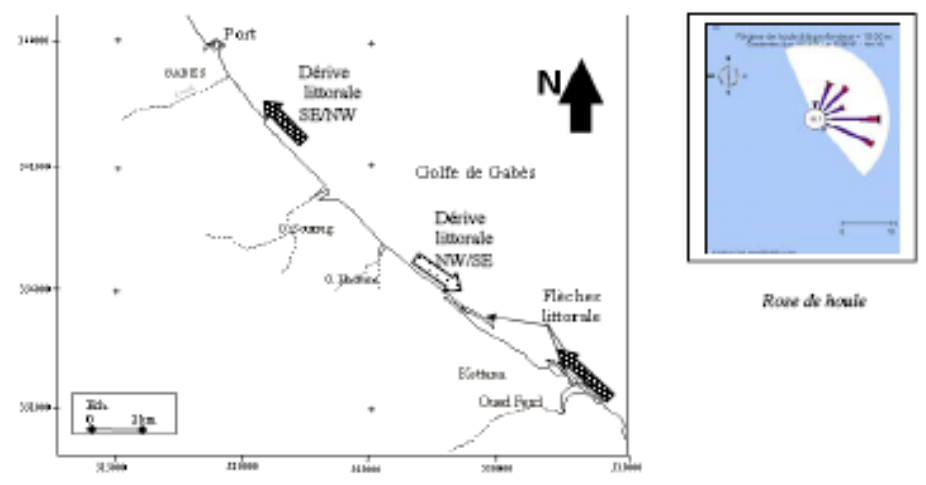

Figure1. Localisation du littoral de Gabès-Oued Ferd (D’après photographie aérienne de 1997)

\section{Résultat et discussion}

La variation du trait de côte entre 1963 et 1997 montre que l'ensemble du rivage est en accrétion, surtout au sud du littoral étudié. En effet au niveau de cette partie sud se jettent deux principaux oueds (l'oued Sourrag et l'oued Ferd) ayant des bassins versants très étendus, dont la superficie atteint respectivement $340 \mathrm{~km}^{2}$ et $400 \mathrm{~km}^{2}$. Ces derniers alimentent ces cours d'eau avec un volume considérable de sédiments qui participent à l'alimentation de la côte lors des crues. En 1973, on a enregistré un apport détritique par l'oued Sourrag de l'ordre de $5616.10^{3} \mathrm{~m}^{3}$ et de $10958.10^{3} \mathrm{~m}^{3}$ par l'oued Ferd (BOURGES, 1974), ce qui a provoqué l'engraissement du littoral durant la période de 1963 à 1985 et donc l'avancée du trait de côte (Fig. 2). De même, de 1985 à 1997 (Fig. 3), le trait de côte a continué de prograder sous l'influence des apports détritiques 
fluviatiles lors des crues de 1990 et 1995 où les précipitations ont été respectivement $380 \mathrm{~mm}$ et $550 \mathrm{~mm}$. Bien que le climat dans la région de Gabès soit de type semi-aride, il est marqué par des événements de forte précipitation. Les pluies sont torrentielles (BEN OUEZDOU, 1983), et donc à l'origine d'importants apports de sédiments détritiques jusqu'à la côte. Ces derniers, en arrivant dans les zones d'embouchure, sont pris en charge par les courants littoraux qui contribuent à l'évolution du trait de côte durant ces années.
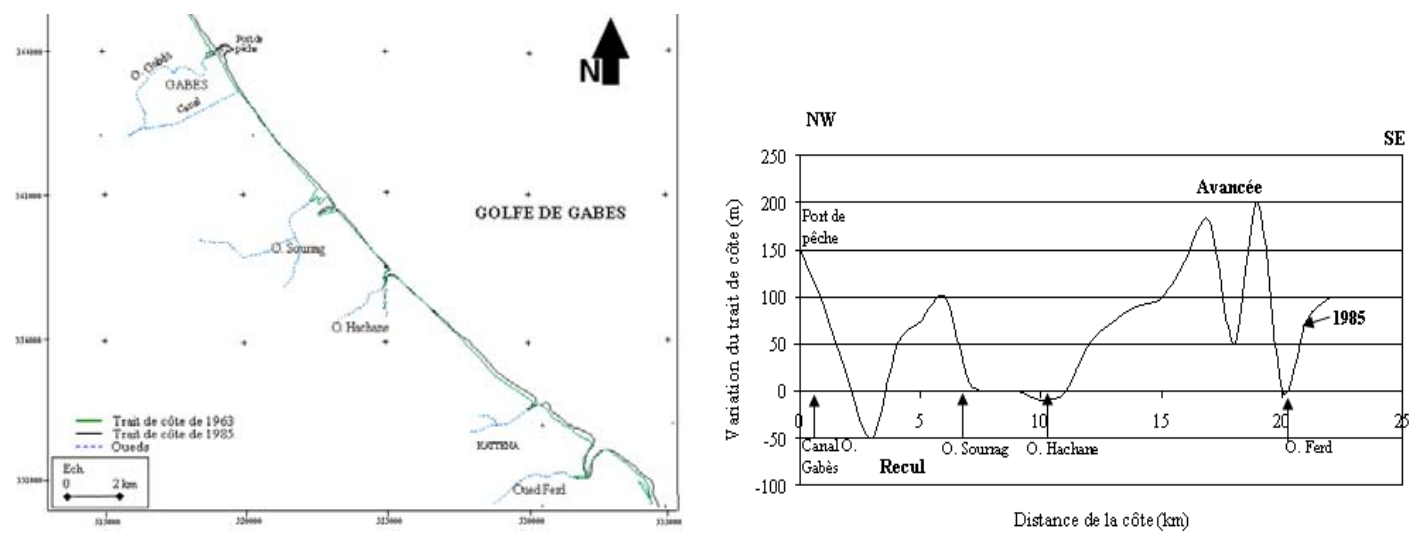

Fig. 2. Variation du trait de côte de la frange littorale (Gabès-O. Ferd) de 1963 à 1985.
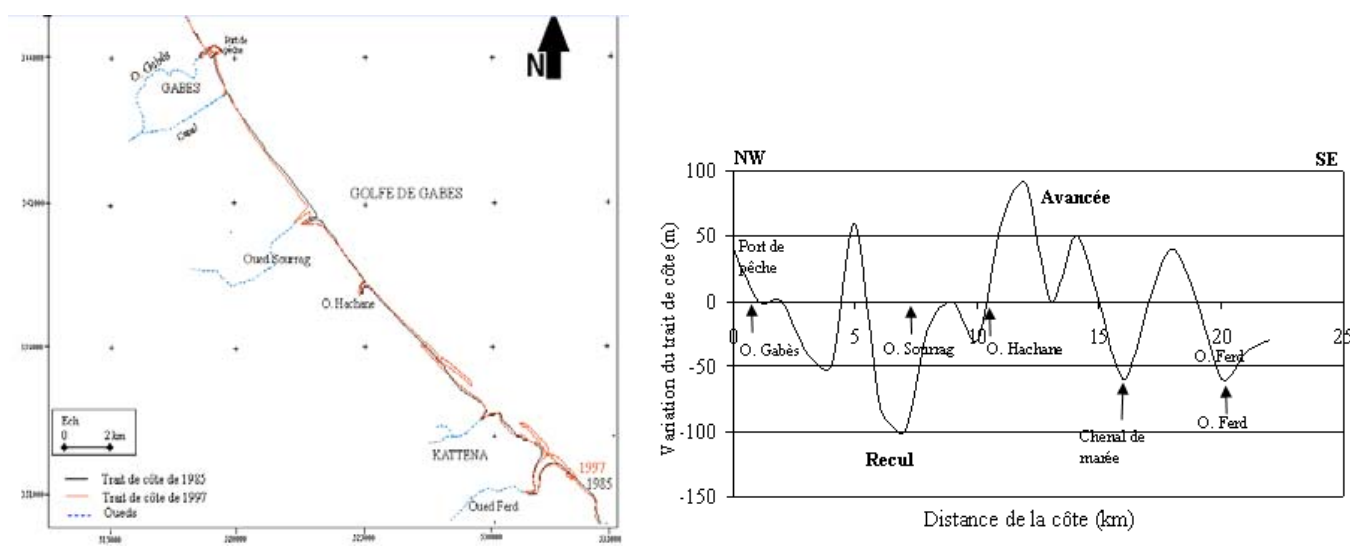

Fig. 3. Variation du trait de côte de la frange littorale (Gabès-O. Ferd) de 1985 à 1997.

En outre, on note l'apparition récente des flèches littorales qui n'existaient pas avant 1985 (Fig. 2 et 3). Nous observons deux flèches de part et d'autre de l'oued Ferd, et celle située au sud de l'oued Sourrag s'étend sur environ plus de $2 \mathrm{~km}$; cette morphologie existe jusqu'à nos jours. La formation et l'évolution de ces flèches littorales sont contrôlées par les courants de dérive littorale de direction NW-SE et SENW, ils s'allongent dans le sens de la dérive selon la direction des houles (Bardi et al., 2010). D’autre part, la partie nord de ce littoral, depuis oued Gabès jusqu'au oued Sourrag, présente globalement un recul du trait de côte observée surtout durant la 
La connaissance de la Mer :

un vecteur du développement durable en Méditerranée

période de 1985 à 1997 (Fig. 3). En effet, l'oued Gabès, possède un bassin versant restreint $\left(88 \mathrm{~km}^{2}\right)$, de plus l'aménagement d'un canal de déviation de l'aval de l'oued à réduit les apports fluviatiles à la côte. À l'abri de la jetée sud du port, nous avons distingué un engraissement et une avancée du trait de côte qui dépasse les 40 m (Fig. 3), qui sont le résultat de l'accumulation des sables par les courants de dérive provenant du SE vers le NW. Cette partie du littoral est en continuelle progradation puisque la partie sud de la jetée du port est de plus en plus ensablée et la largeur de la plage s'étend de jour en jour.

\section{Conclusion}

L'analyse de l'évolution du trait de côte, entre 1963 et 1997, a montré que le littoral depuis le port de Gabès jusqu'à oued Ferd, est assujetti à une dynamique sédimentaire à la suite de l'association de facteurs à la fois anthropiques et hydrodynamiques. On assiste surtout à la formation des flèches littorales et à l'engraissement du littoral essentiellement dans la partie sud. Ces faits sont causés d'une part, par les apports sédimentaires continentaux en provenance de l'arrière pays, apportés par les oueds Ferd et Sourrag lors des périodes de crue, d'autre part, par les courants de dérives littorales qui transitent les sédiments fins le long du littoral du SE vers le NW et/ou de NW vers le SE, et ce suivant la direction de la houle incidente.

\section{Références bibliographiques}

BARDI I. (2010). Morphodynamique de la côte sableuse microtidale à barres le long de la frange littorale Gabès-Oued Ferd, (Golfe de Gabès-Tunisie). Thèse de doctorat, Faculté des sciences de Tunis, Université Tunis El Manar, 202 p.

BARDI I., SOUAYED M., ABDELJAOUAD S. GHARBI W. et SOUISSI R. (2010). Evolution morphodynamique de la côte sableuse microtidale dans la partie sud du Golfe de Gabès : Cas de l'estuaire de l'oued Ferd (Sud de la Tunisie). XI ${ }^{\mathrm{èmes}}$ JNGCGC, France, Ed ${ }^{\circ}$ 11, pp 163-170. doi:10.5150/jngcgc.2010.020-B

BEN OUEZDOU H. (1983). Etude morphologique et stratigraphiques des formations quaternaires dans les alentours du Golfe de Gabès. Thèse de spécialité. Univ. de Tunis II. 220 p.

BOURGES J. (1974). Aperçu sur l'hydrologie du centre Sud tunisien, réseau d'observation et crues exceptionnelles. Rapp. Int. D.R.E., 74 p.

DOLAN R. HAYDEN B., HEYWOOD J. (1978). A new photogrammetric method for determining shoreline erosion. Coastal Engineering, 2, pp 21-39. doi:10.1016/03783839(78)90003-0

THIELER E.R., DANFORTH W.W. (1994). Historical shoreline mapping (II): application of the digital shoreline mapping and analysis systems to shoreline change mapping in Puerto Rico. Journal of Coastal Research, 10 (3), pp 600-620. 